\title{
Micron Thick Colloidal Quantum Dot Solids
}

2 James Z. Fan," Maral Vafaie," Koen Bertens," Mykhailo Sytnyk, Joao M. Pina, Laxmi Kishore Sagar, ${ }_{3}$ Olivier Ouellette, Andrew H. Proppe, Armin Sedighian Rasouli, Yajun Gao, Se-Woong Baek, Bin Chen, ${ }_{4}$ Frédéric Laquai, Sjoerd Hoogland, F. Pelayo García de Arquer, Wolfgang Heiss, and Edward H. Sargent*

Cite This: https://dx.doi.org/10.1021/acs.nanolett.0c01614

5 ABSTRACT: Shortwave infrared colloidal quantum dots (SWIR6 CQDs) are semiconductors capable of harvesting across the 7 AM1.5G solar spectrum. Today's SWIR-CQD solar cells rely on 8 spin-coating; however, these films exhibit cracking once thickness 9 exceeds $\sim 500 \mathrm{~nm}$. We posited that a blade-coating strategy could 10 enable thick QD films. We developed a ligand exchange with an 11 additional resolvation step that enabled the dispersion of SWIR12 CQDs. We then engineered a quaternary ink that combined high13 viscosity solvents with short QD stabilizing ligands. This ink, blade-

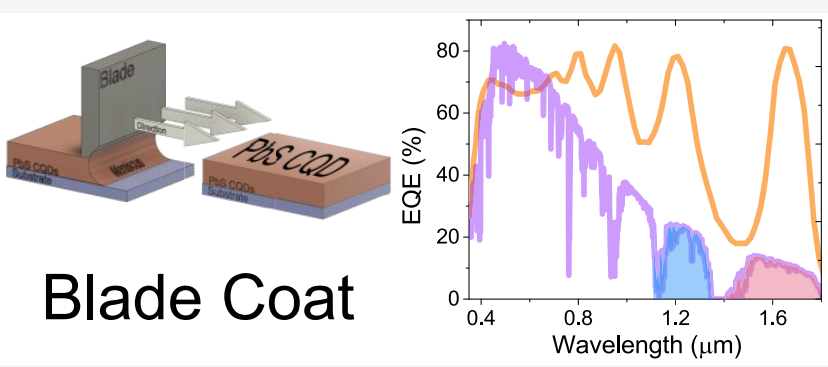
14 coated over a mild heating bed, formed micron-thick SWIR-CQD

15 films. These SWIR-CQD solar cells achieved short-circuit current densities (Jsc) that reach $39 \mathrm{~mA} \mathrm{~cm}^{-2}$, corresponding to the 16 harvest of $60 \%$ of total photons incident under AM1.5G illumination. External quantum efficiency measurements reveal both the first 17 exciton peak and the closest Fabry-Perot resonance peak reaching approximately $80 \%$-this is the highest unbiased EQE reported 18 beyond $1400 \mathrm{~nm}$ in a solution-processed semiconductor.

19 KEYWORDS: infrared photovoltaics, quantum dots, ligand exchange, blade coating

\section{$20 \square$ INTRODUCTION}

21 Shortwave infrared (SWIR) semiconductors are materials 22 capable of absorbing light between 1000 to $3000 \mathrm{~nm}$. This 23 region is of interest for applications including photodetection, 24 light emission, and solar harvesting. ${ }^{1}$ Operating photodetectors 25 in the SWIR spectral range allows for the detection of light 26 transmitted through biological (such as skin), atmospheric 27 (such as water and $\mathrm{CO}_{2}$ ), and synthetic materials (such as 28 plastics). ${ }^{2,3}$ Similarly, SWIR light emitting diodes (LEDs) are 29 used in a variety of applications such as optical communica30 tions and night vision. ${ }^{4,5}$ Finally, SWIR harvesting solar cells 31 are excellent back cell candidates for visible absorbers such as 32 silicon or metal halide perovskites. ${ }^{6}$ Materials such as Ge, 33 InGaAs, and $\mathrm{HgCdTe}$ alloys are excellent SWIR absorbers; 34 however, their epitaxial incompatibility with silicon and flexible 35 electronics and photovoltaics motivate the search for a new 36 generation of materials. $^{7-9}$

37 Colloidal quantum dots (CQDs) are a class of thin film 38 semiconducting materials that can be synthesized in the 39 solution phase. ${ }^{10}$ Their band gap can be tuned to absorb more 40 widely across the AM1.5G spectrum. In particular, SWIR${ }_{41}$ CQDs have been used to fabricate LEDs, photodetectors, and 42 solar cells. ${ }^{11-14}$ SWIR-CQD LEDs exhibit electroluminescence 43 varying from 950 to $1650 \mathrm{~nm}$, and their electroluminescence 44 power is comparable to that of InGaAsP LEDs. ${ }^{15}$ SWIR-CQD 45 photodetectors have reached a detectivity of $10^{12}$ Jones at 2 $\mu$ m. ${ }^{16}$ SWIR-CQD solar cells have exhibited appreciable 46 external quantum efficiencies up to $1800 \mathrm{~nm} .^{17}$

In recent years, research interest has increased in the 48 optimization of SWIR-CQD PV for tandem integration with 49 wide band gap front cells. ${ }^{14,17-27}$ Infrared solar cell metrics 50 such as IR-open circuit voltage (IR-Voc), IR-short circuit 51 current density (IR-Jsc), IR-fill factor (IR-FF), and IR-power 52 conversion efficiency (IR-PCE) are for solar cell operation 53 through a simulated $1100 \mathrm{~nm}$ long pass filter. Innovations in 54 SWIR-CQD synthesis and ligand engineering lead to improve- 55 ments in IR power conversion efficiency (IR-PCE) metrics 56 such as IR-open circuit voltage (IR-Voc) ${ }^{17,28}$ However, the IR- 57 short circuit current densities (IR-Jsc) of IR devices remain 58 low due to inadequate absorption in the IR region.

Improving absorbance in the SWIR region requires 60 increasing the thickness of the absorbing layer and broadening 61 the absorption range of the semiconductor. Both aspects will 62 significantly increase the maximum IR-Jsc, a main contributor 63 for improving IR-PCE. The total available AM1.5G Jsc for 1.164 and $0.7 \mathrm{eV}$ semiconductors are $44.23 \mathrm{~mA} \mathrm{~cm}^{-2}$ and $60.70 \mathrm{~mA} 65$

Received: April 14, 2020

Revised: June 12, 2020

Published: June 16, 2020 
a

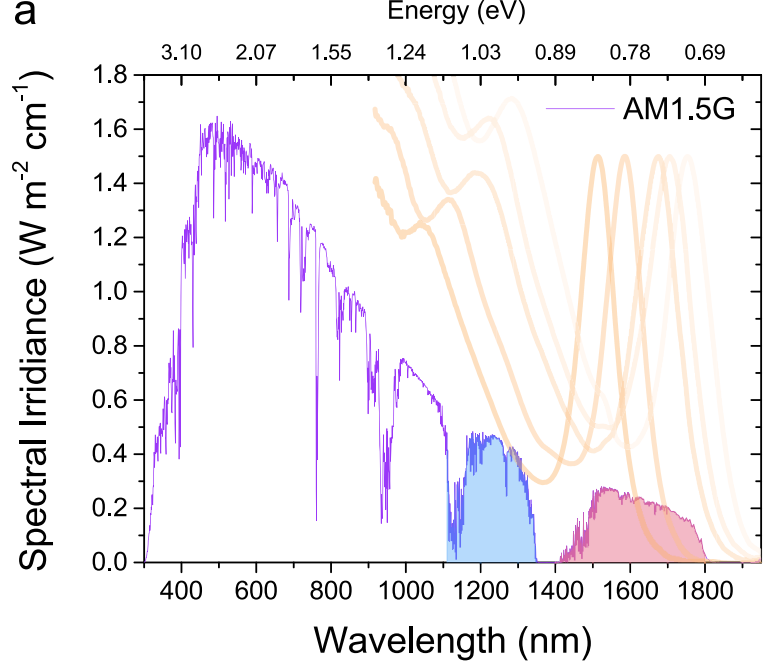

b

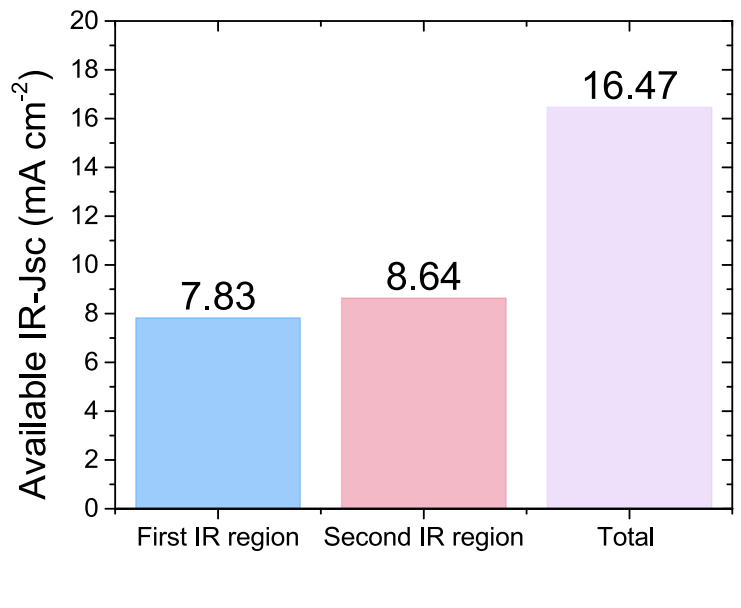

Figure 1. CQDs needed for full AM1.5G harvesting. Absorbance of shortwave infrared quantum dots overlaid on top of the AM1.5G to show potential IR harvesting (a). Available IR-short circuit current densities in the infrared region of the AM1.5G spectrum (b).

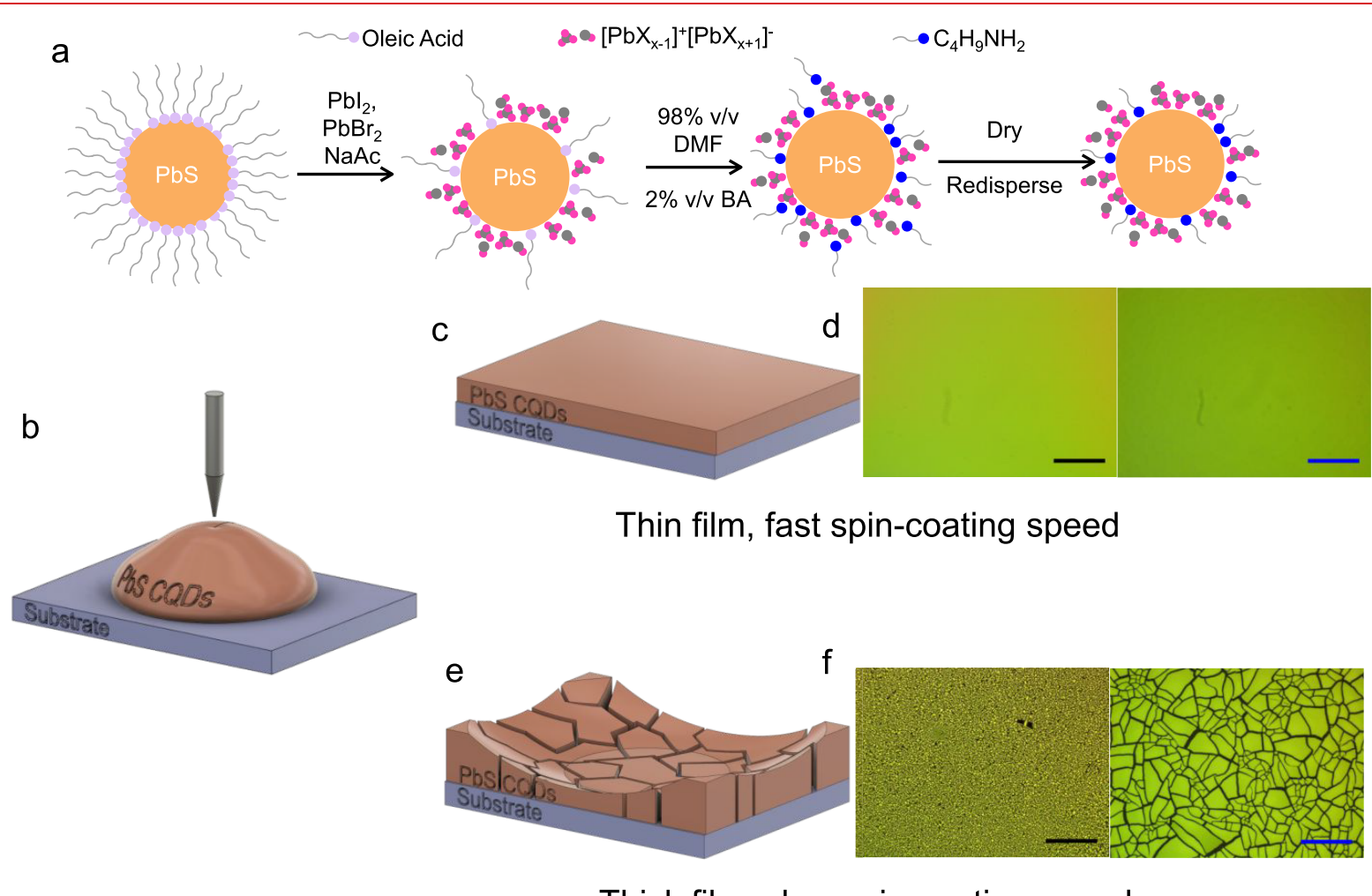

Thick film, slow spin-coating speed

Figure 2. Ligand exchange and film processing. Ligand exchange for SWIR-CQDs with a double precipitation step (a); initial spin-coating schematic; (b) smooth thin film schematic enabled by rapid spin-coating (c); microscope image of the thin spin-coated film (d); cracked thick film schematic enabled by slow spin-coating (e); microscope image of the thick spin-coated film (f). Black scale bars represent $500 \mu \mathrm{m}$. Blue scale bars represent $50 \mu \mathrm{m}$.

$66 \mathrm{~cm}^{-2}$, respectively. ${ }^{29}$ Therefore, this only results in a $37 \%$ ${ }_{67}$ increase in total Jsc. However, the visible wavelengths are 68 mostly absorbed by the front cell; therefore, only the IR ${ }_{69}$ regions are of interest for tandem back cells. Harvesting the 70 first IR-region between $1.1 \mathrm{eV}(\sim 1.1 \mu \mathrm{m})$ to $0.9 \mathrm{eV}(\sim 1.4 \mu \mathrm{m})$ ${ }_{71}$ results in an IR-Jsc of $7.83 \mathrm{~mA} \mathrm{~cm}{ }^{-2}$, while harvesting only the 72 second IR-region between $0.9 \mathrm{eV}(\sim 1.4 \mu \mathrm{m})$ to $0.7 \mathrm{eV}(\sim 1.8$ $73 \mu \mathrm{m})$ results in an IR-Jsc of $8.64 \mathrm{~mA} \mathrm{~cm}{ }^{-2}$. By successfully 74 harvesting both IR regions, devices can reach an IR-Jsc of
$16.47 \mathrm{~mA} \mathrm{~cm}^{-2}$ : a doubling of Jsc (Figure 1a, b). By plotting $75 \mathrm{fl}$ several SWIR-CQD solution absorbance overlapping with the 76 AM1.5G spectrum, we observe that the SWIR-CQDs ranging 77 between $\lambda_{\text {exciton }}=\sim 1500 \mathrm{~nm}$ and $\lambda_{\text {exciton }}=\sim 1750 \mathrm{~nm}$ are 78 capable of harvesting both IR regions. Prior work has 79 demonstrated external quantum efficiencies (EQEs) that 80 reached $80 \%$ in the first IR region. ${ }^{25}$ In addition, EQEs have 81 reached greater than 50\% above the second IR region; 82 however, their nearest Fabry-Perot resonance peak over- 83 


\section{a}

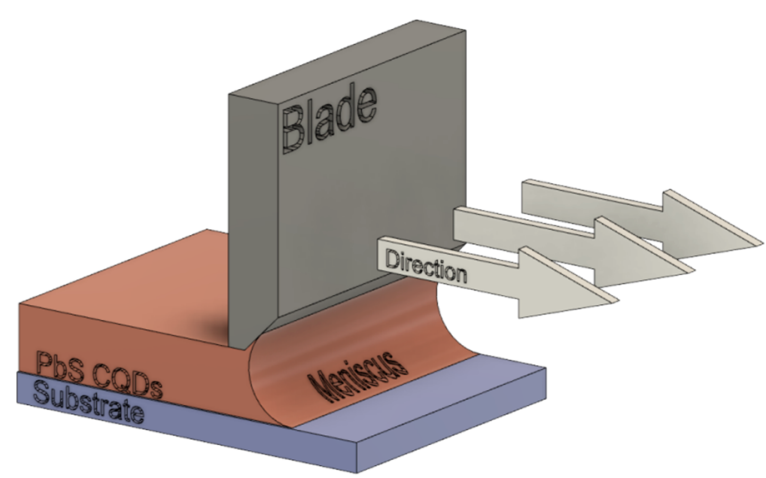

b

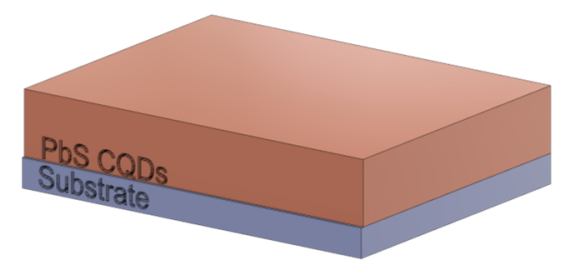

C
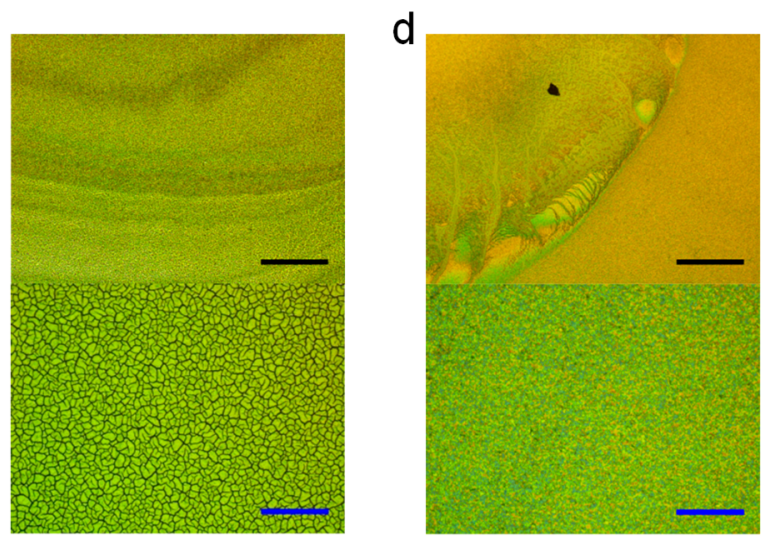

e
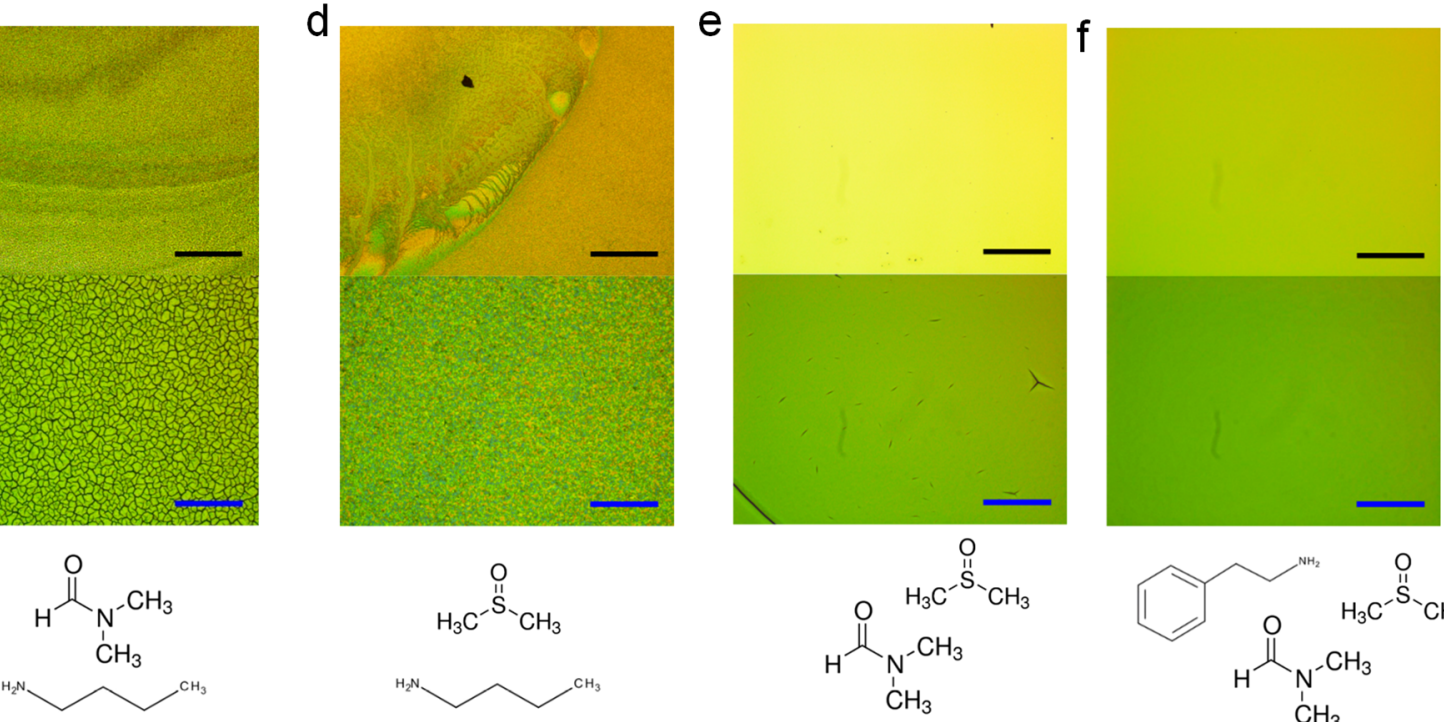

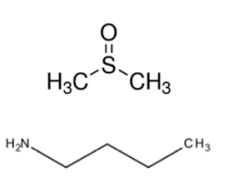

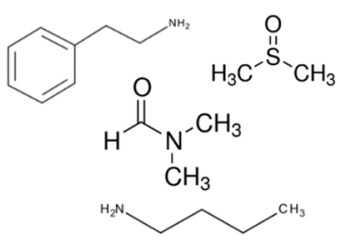

Figure 3. Blade-coating of SWIR CQDs. Blade-coating schematic (a, b). Microscope images of films made with blade-coating solvents composed of DMF and BA (c); DMSO and BA (d); DMF, DMSO, and BA (e); DMF, DMSO, PEA, and BA (f). Black scale bars represent $500 \mu \mathrm{m}$. Blue scale bars represent $50 \mu \mathrm{m}$.

84 lapped detrimentally with the first IR region, causing losses in $85 \mathrm{Jsc}^{23}$

86 Here we demonstrate full AM1.5G harvesting SWIR-CQD 87 solar cells that are capable of harvesting $\sim 60 \%$ of all available 88 solar photons. ${ }^{29}$ We achieve this by implementing the 89 combination of a new ligand exchange, solvent engineering, 90 and blade-coating strategy. The new solvent engineering and 91 blade-coating strategy allows the formation of smooth micron92 thick CQD active layers. The combination of all three 93 techniques results in CQD solar cells achieving the thickest 94 reported PbS-CQD solar cell capable of reaching $80 \%$ external 95 quantum efficiency with both the first exciton peak and nearest 96 Fabry-Perot resonance peak in the infrared region between 971100 and $1800 \mathrm{~nm}-\mathrm{a}$ feat that has not been achieved in the 98 field of QD solar cells.

\section{RESULTS AND DISCUSSION}

100 The ligand exchange and ink deposition for single step SWIR101 CQDs was previously challenging for the CQD community, as 102 these QDs behave much differently than ligand exchanges 103 optimized for single junction harvesting QDs (SJ-QDs, $\lambda_{\text {exciton }}$ $104=900$ to $1000 \mathrm{~nm}$ ). ${ }^{20}$ The specific procedure is described in 105 the Supporting Information, but key differences for processing 106 SJ-QDs and SWIR-CQDs are discussed here. SWIR-CQDs 107 agglomerate immediately following phase transfer from the nonpolar octane phase to the halide-rich DMF phase, while SJ- 108 QDs retain their colloidal stability. It is known that larger 109 diameter SWIR-CQDs have higher amounts of $\mathrm{Pb}(100)$ facets 110 than SJ-QDs. ${ }^{30}$ As a consequence, these SWIR-CQDs are less 111 colloidally stabilized by lead halides in the DMF phase. After 112 the ligand exchange, several modifications must be made to 113 successfully disperse the QDs into a concentrated ink. For the 114 SJ-QD procedure, the exchanged QDs are first destabilized in 115 the DMF phase with an antisolvent such as toluene. Then, the 116 SJ-QDs are centrifuged, dried into a powder, and then 117 dispersed into a solvent such as butylamine (BA). Translating 118 this procedure for SWIR-CQDs does not work, as the SWIR- 119 CQD powders do not disperse in butylamine, preventing film 120 formation.

To overcome this issue, we implemented a double- 122 precipitation step to enable the redispersion of the SWIR- 123 CQDs (Figure 2a). After the completion of the ligand $124 \mathrm{fz}$ exchange procedure, the destabilized SWIR-CQDs were 125 centrifuged and the supernatant was discarded. Then, the 126 moist QDs were first resolvated in a solution of 98:2 v/v 127 DMF/BA mixture. The low amount of BA was needed to 128 stabilize the otherwise aggregated CQDs by exchanging the 129 remaining oleic acid bound to the surface. The toluene 130 antisolvent was added to the solution, and then the mixture 131 was centrifuged. After the supernatant was discarded, the 132 
133 SWIR-CQDs were dried and ready for redispersion. It is not 134 possible to combine the phase transfer ligand exchange and 135 resolvation process into one step. This is because adding the $136 \mathrm{OA}-\mathrm{PbS} \mathrm{CQD}$ s directly into a mixture of a concentrated ligand 137 exchange solution containing a high percentage of redispersion 138 solvent such as butylamine will not allow the efficient phase 139 separation of octane and DMF. This will result in excess oleic 140 acid to disperse in the single mixture, making blade or spin 141 coating result in poor quality films for optoelectronic device 142 fabrication.

143 Thermogravimetric analysis (TGA) revealed the reasoning 144 for the double precipitation step (Supporting Figure S1a). 145 After the first centrifuge, the QDs retained a lot of organic 146 content on the surface of the QD, as indicated by the faster 147 weight loss with increasing temperature. This is attributed to 148 excess oleic acid still bound to the QDs. Since these QDs are 149 passivated by both ionic salts and nonpolar species, these 150 SWIR-CQDs fail to redisperse in traditional QD solvents. After 151 the resolvation step, the organic content on the surface of the 152 QDs significantly dropped, making these QDs mostly 153 passivated with ionic species. Fourier transform infrared 154 (FTIR) spectroscopy characterization on these singly 155 precipitated SWIR-CQD films-materials that dispersed 156 poorly in butylamine-further confirm the presence of organic 157 residue (Supporting Figure S1b, S1c). The signals between 158 1200-1500 and $1080 \mathrm{~cm}^{-1}$ match closely those of OA-PbS $159 \mathrm{CQDs}$ and $-\mathrm{NH}$ bends from free butylamine, respectively.

160 A singular solvent approach, such as the use of butylamine, 161 does not disperse the SWIR-CQDs, and attempting to deposit 162 this ink leads to QD clusters over the film, making this 163 unsuitable for devices (Supporting Figure S2). Therefore, 164 SWIR-CQDs need to be dispersed in a combination of 165 butylamine and DMF. We empirically found that a $30 \%$ 166 mixture of DMF in butylamine was sufficient to create a stable 167 SWIR-CQD colloid, similar to that of previous reports. ${ }^{19,21,22}$ 168 This solvent composition is adequate for spin-coating; the high 169 amount of butylamine allows the majority of solvent to 170 evaporate during the spin-coating process. Spin-coating the 171 QDs at high speeds (>1000 rpm (RPM)) allowed for the 172 formation of thin, smooth films (Figure $2 \mathrm{c}, \mathrm{d}$ ). However, this 173 solvent composition only allows the formation of films up to $174 \sim 500 \mathrm{~nm}$. This thickness is inadequate for infrared absorption, 175 decreasing the overall performance of the solar cell. Spin176 coating at lower speeds (e.g., less than $500 \mathrm{rpm}$ ) does not work 177 to achieve thicker films. As the QD ink reaches the edge of the 178 film, adhesive forces pin the ink to the corners, resulting in a 179 high QD concentration at the corners. As the solvent 180 evaporates, the films crack resulting in uneven, cracked, 181 macroscopically rough films (Figure 2e, f). These pinhole182 containing films result in shorting of the device; thus, single183 step spin-coated QD devices are limited, in practice, to a 184 thickness of ca. $\sim 500 \mathrm{~nm}$.

185 Consequently, we contemplated fabrication techniques such 186 as spray coating, roll-to-roll printing, and doctor-blading to 187 create thicker films. ${ }^{8,31-33}$ Doctor-blading in particular is 188 known for fabricating thick solution-processed perovskite solar 189 cells (e.g., $>1000 \mathrm{~nm}$ films). ${ }^{34}$ However, the full potential for 190 doctor-blading has not yet been exploited in CQD solar cells, 191 as the PCEs of reported doctor-bladed solar cells still remain 192 below their spin-coated counterparts. ${ }^{35}$ In our work, we fully 193 rework our CQD ink conditions for doctor-blading to enable 194 thick film fabrication.
In the blade-coating process, the CQD ink was first 195 deposited on the blade edge to form a meniscus between the 196 substrate and the blade edge. The blade is subsequently 197 dragged over the substrate, as shown in Figure 3 a, b. We $198 \mathrm{f}_{3}$ blade-coated at high speeds $(>25 \mathrm{~mm} / \mathrm{s})$ to enable film 199 formation in the Landu-Levich regime. ${ }^{34,36}$ After the initial 200 coat, the QD ink was still wet on the substrate; therefore, 201 additional heating was needed to evaporate off the excess 202 solvent. To ensure uniform film formation, we performed this 203 blade-coating process on a glass heat bed to enable even 204 evaporation of the film. Without the heat bed, the resulting 205 films contained island-like spots, but by using an underlying 206 heat source, the film underwent an initial solidification which 207 prevented further migration of the ink upon the substrate. ${ }^{33} 208$ We initially tested blade coating with the standard dispersion 209 of 30/70 DMF/BA; however, we noticed that the solvent dried 210 too quickly and formed cracked films under the spin-coat 211 optimized concentrations of ink $\left(>250 \mathrm{mg} \cdot \mathrm{mL}^{-1}\right)$ (Figure $\left.3 \mathrm{c}\right) .212$ At lower concentrations, we noticed that the film dried 213 nonuniformly, resulting in a rough, macroscopic texture across 214 the top of the film. In order to create a thick uniform film, we 215 attempted to engineer the ink with higher boiling point 216 solvents to slow down the evaporation of the film. We first 217 sought to use a combination of dimethyl sulfoxide (DMSO) 218 (boiling point $=189{ }^{\circ} \mathrm{C}$ ) and butylamine to increase the 219 boiling point of the colloid. However, this mixture did not 220 properly disperse the SWIR-CQDs, and thus a PV-grade film 221 cannot be made (Figure 3d). By combining the three solvents 222 \{DMF, DMSO, and BA\}, a stable SWIR-CQD colloid was 223 achieved. This solvent system alone provided improved blade- 224 coated SWIR-CQD films.

225

However, this solvent system caused film cracking when we 226 attempted to fabricate $>1000 \mathrm{~nm}$ films (Figure 3e). Therefore, 227 we incorporated a fourth component, phenethylamine (PEA), 228 to our system. PEA improves the ink mixture for the following 229 reasons: (1) PEA's high boiling point $\left(195{ }^{\circ} \mathrm{C}\right)$ further slows 230 down the evaporation of the SWIR-CQD mixture; (2) PEA's 231 greater than 2-fold viscosity over DMSO strikes a balance 232 between the rate of coating and ink solidification; (3) the 233 primary amine on the PEA stabilizes the lead halide matrix 234 around the SWIR-CQD. ${ }^{35}$ This quaternary solvent system 235 allows us to fabricate smooth QD films thicker than $1000 \mathrm{~nm} 236$ (Figure 3f). Zoomed in microscope images reveal minor 237 differences between the triple solvent and quaternary solvent 238 blade-coated films (Supporting Figure S3). Atomic force 239 microscope (AFM) images also reveal a smooth film for the 240 quaternary ink (Supporting Figure S4). AFM imaging could 241 not be completed for films shown in Figure $3 c$ to $3 \mathrm{e}$ because 242 those films were too rough to be imaged. The fabrication was 243 finalized by an oven annealing step within a controlled 244 atmosphere of nitrogen at $80{ }^{\circ} \mathrm{C}$ for $15 \mathrm{~min}$ to ensure that 245 there was no residual solvent left. Transmission electron 246 microscope (TEM) images reveal that the PbS CQDs remain 247 monodisperse in the quaternary solvent system (Supporting 248 Figure S5).

Additional characterization techniques such as X-ray photo- 250 electron spectroscopy (XPS) and FTIR were used to compare 251 blade-coated and spin-coated films (Supporting Figures S6- 252 S8). FTIR showed similar features for both the spin-coated and 253 blade-coated samples. There are no additional organic peaks 254 seen on the blade-coated films compared to the spin-coated 255 films. The loss of the $\mathrm{C}=\mathrm{C}$ stretch at $\sim 3006 \mathrm{~cm}^{-1}$ is indicative 256 of the successful removal of oleate ligands (Supporting Figure 257 

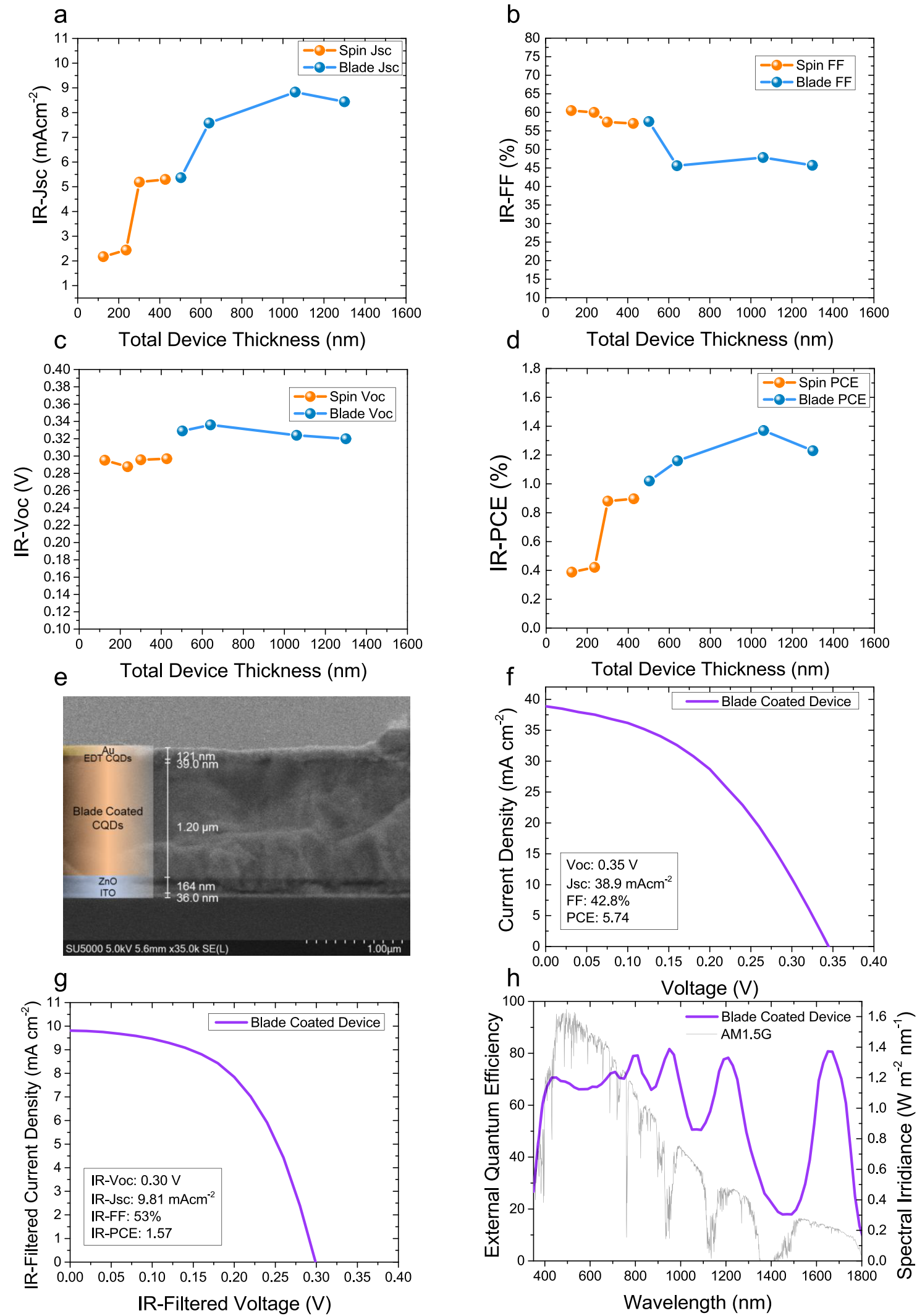

Figure 4. Solar cell metrics. Filtered solar cell parameters with varying thicknesses for IR-Jsc (a), IR-FF (b), IR-Voc (c), and IR-PCE (d). Cross section scanning electron microscope image of a blade-coated device (e). Full spectrum $J-V$ curve of champion blade-coated device (f). IR-filtered $J-V$ curve of champion blade-coated device (g). External quantum efficiency of champion blade-coated device (h).

$258 \mathrm{~S} 8 \mathrm{a}){ }^{37,38}$ The $\mathrm{Pb}$-amine complex is observed at $\sim 3484 \mathrm{~cm}^{-1}$ 259 on both spin-coated and blade-coated films. ${ }^{40}$ The signal 260 between $1240 \mathrm{~cm}^{-1}$ and $1640 \mathrm{~cm}^{-1}$ correspond to the $-\mathrm{NH}$ bends from the $\mathrm{Pb}$-amine complex, similar to a previous 261 report (Supporting Figure S8b). ${ }^{39}$ XPS was used to study the 262 degree of passivation for the SWIR-CQD films (Supporting 263 
264 Figures S6, S7a-d). Blade-coated films showed much higher 265 iodide to sulfur and lead to sulfur ratios, indicting better 266 passivation of the QD. We suspect that the addition of both 267 DMSO and PEA stabilizes residual $\mathrm{PbX}_{2}(\mathrm{X}=\mathrm{I}, \mathrm{Br})$ species on 268 the QD surface, thus increasing the amount of both $\mathrm{I} / \mathrm{S}$ and $269 \mathrm{~Pb} / \mathrm{S}$ ratios. ${ }^{40}$

270 Finally, we fabricated IR harvesting solar cells using both 271 techniques. We used an ITO/ZnO/PbX-PbS/EDT-PbS/Au 272 architecture for the devices. Infrared solar cell metrics such as 273 IR-open circuit voltage (IR-Voc), IR-short circuit current 274 density (IR-Jsc), IR-fill factor (IR-FF), and IR-power 275 conversion efficiency (IR-PCE) were compared for different 276 thicknesses of both spin-coated and blade-coated samples 277 (Figure $4 \mathrm{a}-\mathrm{d}$ ). The PCE metrics under AM1.5G illumination 278 are presented in Supporting Figure S9. A representative cross279 sectional scanning electron microscope (SEM) image reveals a 280 continuous SWIR CQD layer exceeding $1 \mu \mathrm{m}$ (Figure 4e). The 281 active layer thicknesses for spin-coated devices varied between $282 \sim 130$ and $430 \mathrm{~nm}$, while the blade-coated active layer 283 thicknesses ranged from $500 \mathrm{~nm}$ to $1.3 \mu \mathrm{m}$ Our blade-coating 284 solvent was optimized to favor thicker active layers; as a result, 285 blade coating does not produce high-quality sub-500 nm 286 devices. Indeed, fabricating thin blade-coated films require 287 slow coating speeds, which leads to the uneven evaporation of 288 CQD solvent. Even if we did reoptimize our blade-coating QD 289 inks for thinner films, the lower absorbance of the active layer 290 is unfavorable for increasing the overall PCE. As mentioned 291 earlier, spin-coated devices above $500 \mathrm{~nm}$ showed significant 292 signs of cracking; therefore, we were not able to successfully 293 fabricate thicker spin-coated devices.

294 The IR-Jsc of these devices showed an upward increase as 295 the thickness of the active layer increased. This is expected, as 296 the absorbance of the device increases with thickness. Selected 297 external quantum efficiency (EQE) spectra reveal the 298 phenomena for the rapid increase in Jsc (Supporting Figure 299 S10). As expected, the first exciton peak generally increases 300 with active layer thickness. Interestingly, the gap between the 301 first exciton peak and the closest Fabry-Perot resonance peak 302 becomes favorably narrower with increasing active layer 303 thickness. The overlap between the Fabry-Perot resonance 304 peak and the first IR region is mainly responsible for the large 305 increases with Jsc. Jsc does not increase monotonically as seen 306 in other recent studies. ${ }^{17,18,41}$ In our case, when the spin307 coated film increased from ca. 300 to $430 \mathrm{~nm}$, there was a 308 slight drop in Jsc (Supporting Figure S9).

309 The IR-FF decreased with increasing thicknesses, which is a 310 result of increased carrier recombination for thicker devices 311 (Figure $4 \mathrm{~b}$ ). The $J V$ curves of the blade-coated sample show 312 the IR-FF gradually decreasing with thickness (Supporting 313 Figure S11a). In addition, the IR-power density vs voltage 314 curves and IR-maximum power point also shift toward lower 315 voltages with increasing thickness-indicative of the slight 316 decrease in IR-FF (Supporting Figure S11b). Interestingly, the 317 IR-Voc was higher for the blade-coated set. We hypothesized 318 that film formation as a result of blade coating improved the 319 solid state QD dispersity. The absorbance of the blade-coated 320 films showed a narrower fwhm than the spin-coated ones 321 (Supporting Figure S12). Blade-coated films also have a lower 322 film Stokes shift of $19 \mathrm{meV}$ compared to a spin-coated film 323 Stokes shift of $62 \mathrm{meV}$. A lower Stokes shift is indicative of 324 improved film monodispersity, leading to flatter energy 325 landscapes and higher built-in potentials. ${ }^{14,42}$ The champion $326 \mathrm{~J}-V$ curve under full AM1.5G illumination has a Jsc of 38.9
$\mathrm{mAcm}^{-2}$, currently the highest Jsc for all solution processed, 327 thin film solar cells (Figure 4f). This Jsc value also accounts for 328 approximately $60 \%$ of total solar photons under AM1.5G 329 illumination. ${ }^{29}$ The IR-Jsc also reaches $9.81 \mathrm{mAcm}^{-2}, 70 \% 330$ greater than that of previously reported IR-CQD solar cells 331 (Figure 4g). ${ }^{17,28}$ The unbiased EQE showed both the first 332 exciton peak at $\sim 1670 \mathrm{~nm}$ reaching $\sim 80 \%$, and the nearest 333 Fabry-Perot resonance peak at $\sim 1210 \mathrm{~nm}$ approaching $80 \% 334$ (Figure 4h). The exciton peak at $1670 \mathrm{~nm}$ exceeds previous 335 CQD EQE exciton peaks by over $30 \%$ and is comparable to 336 EQE values from single crystalline Ge solar cells in this IR 337 region. ${ }^{17,43,44}$ This work presents the highest unbiased EQE for 338 all solution-processed semiconductor ranges beyond $1400 \mathrm{~nm}, 339$ suggesting further applications for IR detection. $3,45,46$

In conclusion, we utilized the full advantage of blade coating 341 to create thick, smooth CQD films. Previous spin-coating 342 methods caused significant cracking upon attempting to 343 increase thickness. By engineering a four-component high 344 boiling viscous CQD ink, we could readily fabricate micron 345 thick $\mathrm{PbS}$ CQD active layers. These thick layers offer 346 incredible improvements in IR-harvesting mainly due to the 347 favorable matching of both the first exciton peak and nearest 348 Fabry-Perot peak to the AM1.5G spectrum. These blade- 349 coated CQD devices harvest the entire solar spectrum, 350 providing one more step toward the fabrication of triple and 351 quad-junction solar cells.

\section{ASSOCIATED CONTENT}

The Supporting Information is available free of charge at 355 https://pubs.acs.org/doi/10.1021/acs.nanolett.0c01614. 356

Experimental Methods, TGA, microscope images, XPS, 357 $J-V$ curves, EQE spectra, absorbance, and PL data 358 (PDF)

\section{AUTHOR INFORMATION}

Edward H. Sargent - Department of Electrical and Computer 362 Engineering, University of Toronto, Toronto, Ontario M5S 3G4, 363 Canada; (1) orcid.org/0000-0003-0396-6495; 364 Email: ted.sargent@utoronto.ca

Authors

James Z. Fan - Department of Electrical and Computer 367 Engineering, University of Toronto, Toronto, Ontario M5S 3G4, 368 Canada; (1) orcid.org/0000-0002-1594-865X

Maral Vafaie - Department of Electrical and Computer 370 Engineering, University of Toronto, Toronto, Ontario M5S 3G4, 371 Canada; (1) orcid.org/0000-0001-9119-6499 372

Koen Bertens - Department of Electrical and Computer 373 Engineering, University of Toronto, Toronto, Ontario M5S 3G4, 374 Canada

Mykhailo Sytnyk - Department of Materials Science and 376 Engineering, Friedrich-Alexander-Universität Erlangen- 377 Nürnberg, Nürnberg 90429, Germany 378

Joao M. Pina - Department of Electrical and Computer 379 Engineering, University of Toronto, Toronto, Ontario M5S 3G4, 380 Canada; orcid.org/0000-0002-3448-0028

Laxmi Kishore Sagar - Department of Electrical and Computer 382 Engineering, University of Toronto, Toronto, Ontario M5S 3G4, 383 Canada; orcid.org/0000-0002-7656-7308 
385 Olivier Ouellette - Department of Electrical and Computer Engineering, University of Toronto, Toronto, Ontario M5S 3G4, Canada; (1) orcid.org/0000-0001-5708-5058

Andrew H. Proppe - Department of Electrical and Computer Engineering, University of Toronto, Toronto, Ontario M5S 3G4, Canada; (1) orcid.org/0000-0003-3860-9949

Armin Sedighian Rasouli - Department of Electrical and Computer Engineering, University of Toronto, Toronto, Ontario M5S 3G4, Canada

Yajun Gao - King Abdullah University of Science and Technology (KAUST), KAUST Solar Center (KSC), Physical Sciences and Engineering Division (PSE), Material Science and Engineering Program (MSE), Thuwal 23955-6900, Saudi Arabia

Se-Woong Baek - Department of Electrical and Computer Engineering, University of Toronto, Toronto, Ontario MSS 3G4, Canada

Bin Chen - Department of Electrical and Computer Engineering, University of Toronto, Toronto, Ontario MSS 3G4, Canada

Frédéric Laquai - King Abdullah University of Science and Technology (KAUST), KAUST Solar Center (KSC), Physical Sciences and Engineering Division (PSE), Material Science and Engineering Program (MSE), Thuwal 23955-6900, Saudi Arabia; (1) orcid.org/0000-0002-5887-6158

Sjoerd Hoogland - Department of Electrical and Computer Engineering, University of Toronto, Toronto, Ontario MSS 3G4, Canada

F. Pelayo García de Arquer - Department of Electrical and Computer Engineering, University of Toronto, Toronto, Ontario M5S 3G4, Canada; orcid.org/0000-0003-2422-6234

Wolfgang Heiss - Department of Materials Science and Engineering, Friedrich-Alexander-Universität ErlangenNürnberg, Nürnberg 90429, Germany; 이이.orido0000003-0430-9550

419 Complete contact information is available at:

420 https://pubs.acs.org/10.1021/acs.nanolett.0c01614

\section{Author Contributions}

422 IJ.Z.F., M.V., and K.B. contributed equally

\section{Funding}

424 This work was supported by Ontario Research Fund-Research 425 Excellence program (ORF7 ministry of Research and 426 Innovation, Ontario Research Fund-Research Excellence 427 Round 7) and by the Natural Sciences and Engineering 428 Research Council (NSERC) of Canada in the form of 429 Alexander Graham Bell Canada Graduate Scholarships 430 (CGS-D), Materials for Enhanced Energy Technologies 431 (MEET) scholarships, and the NSERC Collaborative Research 432 and Training Experience (CREATE) Program Grant Number 433 466083. The authors acknowledge financial support from QD 434 Solar. This publication is also based upon work supported by 435 the King Abdullah University of Science and Technology 436 (KAUST) Office of Sponsored Research (OSR) under Award 437 No. OSR-CRG2018-3737.

438 Notes

439 The authors declare no competing financial interest.

\section{ACKNOWLEDGMENTS}

441 The authors thank L. Levina, E. Palmiano, R. Wolowiec, and 442 D. Kopilovic for their assistance during the period of study. 443 The authors also thank the members of QD Solar for their 444 assistance and discussions during the period of study: V. Tran,
T. Vo, T. Gibbs, M. Labine, A. MacDonald, E. Mosaferi, R. 445 Quintero-Bermudez, and A. Fisher.

446

\section{ABBREVIATIONS}

447

Short wave infrared (SWIR); Colloidal Quantum Dot (CQD); 448 Infrared (IR); Single junction (SJ); Butylamine (BA); 449 Theromgravimetric analysis (TGA); Dimethyl sulfoxide 450 (DMSO); X-ray Photoelectron Spectroscopy (XPS); Fourier 451 Transform Infrared Spectroscopy (FTIR); Phenethylamine 452 (PEA); Scanning electron microscope (SEM); Open circuit 453 voltage (Voc); Short circuit current density (Jsc); Fill factor 454 (FF); Power conversion efficiency (PCE); Full width at half- 455 maximum (fwhm)

456

\section{REFERENCES}

457

(1) García De Arquer, F. P.; Armin, A.; Meredith, P.; Sargent, E. H. 458 Solution-Processed Semiconductors for next-Generation Photodetec- 459 tors. Nat. Rev. Mater. 2017, 2 (3), 1-16.

(2) Wilson, R. H.; Nadeau, K. P.; Jaworski, F. B.; Tromberg, B. J.; 461 Durkin, A. J. Review of Short-Wave Infrared Spectroscopy and 462 Imaging Methods for Biological Tissue Characterization. J. Biomed. 463 Opt. 2015, 20 (3), 030901.

(3) Clifford, J. P.; Konstantatos, G.; Johnston, K. W.; Hoogland, S.; 465 Levina, L.; Sargent, E. H. Fast, Sensitive and Spectrally Tuneable 466 Colloidal-Quantum-Dot Photodetectors. Nat. Nanotechnol. 2009, 4467 (1), 40-44.

(4) Jeon, E. S.; Kim, J. H.; Hong, H. G.; Batchuluun, G.; Park, K. R. 469 Human Detection Based on the Generation of a Background Image 470 and Fuzzy System by Using a Thermal Camera. Sensors 2016, 16 (4), 471 453.

(5) Hansen, M. P.; Malchow, D. S. Overview of SWIR Detectors, 473 Cameras, and Applications. Proc. SPIE 2008, 6939 (March 2008), 474 69390I.

(6) Lumb, M. P.; Mack, S.; Schmieder, K. J.; González, M.; Bennett, 476 M. F.; Scheiman, D.; Meitl, M.; Fisher, B.; Burroughs, S.; Lee, K. T.; 477 et al. GaSb-Based Solar Cells for Full Solar Spectrum Energy 478 Harvesting. Adv. Energy Mater. 2017, 7 (20), 1700345.

(7) Leem, J. W.; Yu, J. S.; Kim, J. N.; Noh, S. K. Theoretical 480 Modeling and Optimization of III-V GaInP/GaAs/Ge Monolithic 481 Triple-Junction Solar Cells. J. Korean Phys. Soc. 2014, 64 (10), 1561- 482 1565.

(8) Kramer, I. J.; Moreno-Bautista, G.; Minor, J. C.; Kopilovic, D.; 484 Sargent, E. H. Colloidal Quantum Dot Solar Cells on Curved and 485 Flexible Substrates. Appl. Phys. Lett. 2014, 105 (16), 163902.

(9) Norton, P. HgCdTe Infrared Detectors. Opto-electronics Rev. 487 2002, 10 (3), 159-174.

(10) Wang, R.; Shang, Y.; Kanjanaboos, P.; Zhou, W.; Ning, Z.; 489 Sargent, E. H. Colloidal Quantum Dot Ligand Engineering for High 490 Performance Solar Cells. Energy Environ. Sci. 2016, 9 (4), 1130-1143. 491

(11) Yan, L.; Shen, X.; Zhang, Y.; Zhang, T.; Zhang, X.; Feng, Y.; 492 Yin, J.; Zhao, J.; Yu, W. W. Near-Infrared Light Emitting Diodes 493 Using PbSe Quantum Dots. RSC Adv. 2015, 5 (67), 54109-54114. 494

(12) Yang, Z.; Voznyy, O.; Liu, M.; Yuan, M.; Ip, A. H.; Ahmed, O. 495 S.; Levina, L.; Kinge, S.; Hoogland, S.; Sargent, E. H. All-Quantum- 496 Dot Infrared Light-Emitting Diodes. ACS Nano 2015, 9 (12), 12327- 497 12333.

(13) Tang, H.; Zhong, J.; Chen, W.; Shi, K.; Mei, G.; Zhang, Y.; 499 Wen, Z.; Müller-Buschbaum, P.; Wu, D.; Wang, K.; et al. Lead Sulfide 500 Quantum Dot Photodetector with Enhanced Responsivity through a 501 Two-Step Ligand-Exchange Method. ACS Appl. Nano Mater. 2019, 2502 (10), 6135-6143.

(14) Ip, A. H.; Kiani, A.; Kramer, I. J.; Voznyy, O.; Movahed, H. F.; 504 Levina, L.; Adachi, M. M.; Hoogland, S.; Sargent, E. H. Infrared 505 Colloidal Quantum Dot Photovoltaics via Coupling Enhancement 506 and Agglomeration Suppression. ACS Nano 2015, 9 (9), 8833-8842. 507

(15) Hyun, B.-R.; Malliaras, G. G.; Wise, F. W.; Hanrath, T.; Choi, J. 508 J.; Sun, L.; Stachnik, D.; Bartnik, A. C. Bright Infrared Quantum-Dot 509 
510 Light-Emitting Diodes through Inter-Dot Spacing Control. Nat. 511 Nanotechnol. 2012, 7 (6), 369-373.

512 (16) Huo, N.; Gupta, S.; Konstantatos, G. MoS2-HgTe Quantum 513 Dot Hybrid Photodetectors beyond 2 Mm. Adv. Mater. 2017, 29 (17), 5141606576 .

515 (17) Bi, Y.; Bertran, A.; Gupta, S.; Ramiro, I.; Pradhan, S.; 516 Christodoulou, S.; Majji, S.; Akgul, Z.; Konstantatos, G. Solution 517 Processed Infrared- and Thermo- Photovoltaics Based on 0.7 EV 518 Bandgap PbS Colloidal Quantum Dots. Nanoscale 2019, 11 (3), 838519843.

520 (18) Sun, B.; Ouellette, O.; de Arquer, F. P. G.; Voznyy, O.; Kim, Y.; 521 Wei, M.; Proppe, A. H.; Saidaminov, M. I.; Xu, J.; Liu, M.; et al. 522 Multibandgap Quantum Dot Ensembles for Solar-Matched Infrared 523 Energy Harvesting. Nat. Commun. 2018, 9 (1), 1-7.

524 (19) Fan, J. Z.; Andersen, N. T.; Biondi, M.; Todorović, P.; Sun, B.; 525 Ouellette, O.; Abed, J.; Sagar, L. K.; Choi, M.; Hoogland, S.; et al. 526 Mixed Lead Halide Passivation of Quantum Dots. Adv. Mater. 2019, 52731 (48), 1904304.

528 (20) Kiani, A.; Sutherland, B. R.; Kim, Y.; Ouellette, O.; Levina, L.; 529 Walters, G.; Dinh, T.; Liu, M.; Voznyy, O.; Lan, X.; et al. Single-Step 530 Colloidal Quantum Dot Films for Infrared Solar Harvesting. Appl. 531 Phys. Lett. 2016, 109 (18), 183105.

532 (21) Fan, J. Z.; Liu, M. M.; Voznyy, O.; Sun, B.; Levina, L.; 533 Quintero-Bermudez, R.; Liu, M. M.; Ouellette, O.; García de Arquer, 534 F. P.; Hoogland, S.; et al. Halide Re-Shelled Quantum Dot Inks for 535 Infrared Photovoltaics. ACS Appl. Mater. Interfaces 2017, 9 (43), 536 37536-37541.

537 (22) Kim, Y.; Che, F.; Jo, J. W.; Choi, J.; García de Arquer, F. P.; 538 Voznyy, O.; Sun, B.; Kim, J.; Choi, M.-J.; Quintero-Bermudez, R.; 539 et al. A Facet-Specific Quantum Dot Passivation Strategy for Colloid 540 Management and Efficient Infrared Photovoltaics. Adv. Mater. 2019, 54131 (17), 1805580.

542 (23) Jo, J. W.; Choi, J.; García De Arquer, F. P.; Seifitokaldani, A.; 543 Sun, B.; Kim, Y.; Ahn, H.; Fan, J.; Quintero-Bermudez, R.; Kim, J.; 544 et al. Acid-Assisted Ligand Exchange Enhances Coupling in Colloidal 545 Quantum Dot Solids. Nano Lett. 2018, 18 (7), 4417-4423.

546 (24) Choi, J.; Jo, J. W.; de Arquer, F. P. G.; Zhao, Y. B.; Sun, B.; 547 Kim, J.; Choi, M. J.; Baek, S. W.; Proppe, A. H.; Seifitokaldani, A.; 548 et al. Activated Electron-Transport Layers for Infrared Quantum Dot 549 Optoelectronics. Adv. Mater. 2018, 30 (29), 1801720.

550 (25) Bi, Y.; Pradhan, S.; Gupta, S.; Akgul, M. Z.; Stavrinadis, A.; 551 Konstantatos, G. Infrared Solution-Processed Quantum Dot Solar 552 Cells Reaching External Quantum Efficiency of $80 \%$ at $1.35 \mathrm{Mm}$ and $553 \mathrm{Jsc}$ in Excess of $34 \mathrm{MA} \mathrm{Cm}-2$. Adv. Mater. 2018, 30 (7), 1704928.

554 (26) Kim, J.; Ouellette, O.; Voznyy, O.; Wei, M.; Choi, J.; Choi, M.; 555 Jo, J. W.; Baek, S.; Fan, J.; Saidaminov, M. I.; et al. Butylamine556 Catalyzed Synthesis of Nanocrystal Inks Enables Efficient Infrared 557 CQD Solar Cells. Adv. Mater. 2018, 30 (45), 1803830.

558 (27) Baek, S. W.; Molet, P.; Choi, M. J.; Biondi, M.; Ouellette, O.; 559 Fan, J.; Hoogland, S.; García de Arquer, F. P.; Mihi, A.; Sargent, E. H. 560 Nanostructured Back Reflectors for Efficient Colloidal Quantum-Dot 561 Infrared Optoelectronics. Adv. Mater. 2019, 31 (33), 1901745.

562 (28) Xia, Y.; Liu, S.; Wang, K.; Yang, X.; Lian, L.; Zhang, Z.; He, J.; 563 Liang, G.; Wang, S.; Tan, M.; et al. Cation-Exchange Synthesis of 564 Highly Monodisperse PbS Quantum Dots from ZnS Nanorods for 565 Efficient Infrared Solar Cells. Adv. Funct. Mater. 2020, 30 (4), 5661907379

567 (29) Rühle, S. Tabulated Values of the Shockley-Queisser Limit for 568 Single Junction Solar Cells. Sol. Energy 2016, 130 (June 2016), 139569147.

570 (30) Choi, H.; Ko, J. H.; Kim, Y. H.; Jeong, S. Steric-Hindrance571 Driven Shape Transition in PbS Quantum Dots: Understanding Size572 Dependent Stability. J. Am. Chem. Soc. 2013, 135 (14), 5278-5281. 573 (31) Kirmani, A. R.; Sheikh, A. D.; Niazi, M. R.; Haque, M. A.; Liu, 574 M.; de Arquer, F. P. G.; Xu, J.; Sun, B.; Voznyy, O.; Gasparini, N.; 575 et al. Overcoming the Ambient Manufacturability-Scalability-Perform576 ance Bottleneck in Colloidal Quantum Dot Photovoltaics. Adv. Mater. 577 2018, 30 (35), 1870260.
(32) Aqoma, H.; Jang, S. Y. Solid-State-Ligand-Exchange Free 578 Quantum Dot Ink-Based Solar Cells with an Efficiency of $10.9 \% .579$ Energy Environ. Sci. 2018, 11 (6), 1603-1609.

580

(33) Choi, M. J.; Kim, Y. J.; Lim, H.; Alarousu, E.; Adhikari, A.; 581 Shaheen, B. S.; Kim, Y. H.; Mohammed, O. F.; Sargent, E. H.; Kim, J. 582 Y.; et al. Tuning Solute-Redistribution Dynamics for Scalable 583 Fabrication of Colloidal Quantum-Dot Optoelectronics. Adv. Mater. 584 2019, 31 (32), 1805886.

(34) Deng, Y.; Zheng, X.; Bai, Y.; Wang, Q.; Zhao, J.; Huang, J. 586 Surfactant-Controlled Ink Drying Enables High-Speed Deposition of 587 Perovskite Films for Efficient Photovoltaic Modules. Nat. Energy 588 2018, 3 (7), 560-566.

(35) Xu, J.; Voznyy, O.; Liu, M.; Kirmani, A. R.; Walters, G.; Munir, 590 R.; Abdelsamie, M.; Proppe, A. H.; Sarkar, A.; García De Arquer, F. 591 P.; et al. 2D Matrix Engineering for Homogeneous Quantum Dot 592 Coupling in Photovoltaic Solids. Nat. Nanotechnol. 2018, 13 (6), 593 456-462.

594

(36) Le Berre, M.; Chen, Y.; Baigl, D. From Convective Assembly to 595 Landau - Levich Deposition of Multilayered Phospholipid Films of 596 Controlled Thickness. Langmuir 2009, 25 (5), 2554-2557.

(37) Zhang, X.; Cappel, U. B.; Jia, D.; Zhou, Q.; Du, J.; Sloboda, T.; 598 Svanström, S.; Johansson, F. O. L.; Lindblad, A.; Giangrisostomi, E.; 599 et al. Probing and Controlling Surface Passivation of $\mathrm{PbS}$ Quantum 600 Dot Solid for Improved Performance of Infrared Absorbing Solar 601 Cells. Chem. Mater. 2019, 31 (11), 4081-4091. 602

(38) Albaladejo-Siguan, M.; Becker-Koch, D.; Taylor, A. D.; Sun, Q.; 603 Lami, V.; Oppenheimer, P. G.; Paulus, F.; Vaynzof, Y. Efficient and 604 Stable PbS Quantum Dot Solar Cells by Triple-Cation Perovskite 605 Passivation. ACS Nano 2020, 14 (1), 384-393.

606

(39) Acik, M.; Alam, T. M.; Guo, F.; Ren, Y.; Lee, B.; Rosenberg, R. 607 A.; Mitchell, J. F.; Park, I. K.; Lee, G.; Darling, S. B. Substitutional 608 Growth of Methylammonium Lead Iodide Perovskites in Alcohols. 609 Adv. Energy Mater. 2018, 8 (5), 1701726.

610

(40) Jeon, N. J.; Noh, J. H.; Kim, Y. C.; Yang, W. S.; Ryu, S.; Seok, S. 611 Il. Solvent Engineering for High-Performance Inorganic-Organic 612 Hybrid Perovskite Solar Cells. Nat. Mater. 2014, 13 (9), 897.

(41) Lin, Q.; Armin, A.; Nagiri, R. C. R.; Burn, P. L.; Meredith, P. 614 Electro-Optics of Perovskite Solar Cells. Nat. Photonics 2015, 9 (2), 615 $106-112$.

616

(42) Liu, M.; Voznyy, O.; Sabatini, R.; García De Arquer, F. P.; 617 Munir, R.; Balawi, A. H.; Lan, X.; Fan, F.; Walters, G.; Kirmani, A. R.; 618 et al. Hybrid Organic-Inorganic Inks Flatten the Energy Landscape in 619 Colloidal Quantum Dot Solids. Nat. Mater. 2017, 16 (2), 258-263. 620

(43) Guter, W.; Schöne, J.; Philipps, S. P.; Steiner, M.; Siefer, G.; 621 Wekkeli, A.; Welser, E.; Oliva, E.; Bett, A. W.; Dimroth, F. Current- 622 Matched Triple-Junction Solar Cell Reaching 41.1\% Conversion 623 Efficiency under Concentrated Sunlight. Appl. Phys. Lett. 2009, 94624 (22), 223504.

(44) Grás, A.; Jüngst, G.; Campesato, R.; Gori, G.; Greco, E. 626 External Quantum Efficiency and First Results of Electric Perform- 627 ance Measurements on a Quadruple Junction Space Solar Cell. In E3S 628 Web of Conferences 2017, 16, 1-4.

(45) Nikitskiy, I.; Goossens, S.; Kufer, D.; Lasanta, T.; Navickaite, 630 G.; Koppens, F. H. L.; Konstantatos, G. Integrating an Electrically 631 Active Colloidal Quantum Dot Photodiode with a Graphene 632 Phototransistor. Nat. Commun. 2016, 7 (May), 1-8. 633

(46) Gao, J.; Nguyen, S. C.; Bronstein, N. D.; Alivisatos, A. P. 634 Solution-Processed, High-Speed, and High-Quantum-Efficiency 635 Quantum Dot Infrared Photodetectors. ACS Photonics 2016, 3 (7), 636 $1217-1222$. 